\title{
BMJ Open Comparing the effects of two spasticity management strategies on the long-term outcomes of individuals with bilateral spastic cerebral palsy: a multicentre cohort study protocol
}

To cite: Munger ME, Chen BPJ, MacWilliams BA, et al. Comparing the effects of two spasticity management strategies on the long-term outcomes of individuals with bilateral spastic cerebral palsy: a multicentre cohort study protocol. BMJ Open 2019;9:e027486. doi:10.1136/ bmjopen-2018-027486

- Prepublication history and additional material for this paper are available online. To view please visit the journal (http:// dx.doi.org/10.1136/bmjopen2018-027486).

Received 24 October 2018 Revised 14 March 2019 Accepted 7 May 2019

D) Check for updates

(c) Author(s) (or their employer(s)) 2019. Re-use permitted under CC BY-NC. No commercial re-use. See rights and permissions. Published by BMJ.

For numbered affiliations see end of article.

Correspondence to Ms Meghan E Munger; meghanemunger@ gillettechildrens.com

\section{ABSTRACT}

Introduction Spasticity is one of the primary pathologies associated with cerebral palsy (CP), yet no definitive evidence exists to guide the appropriate level of spasticity management for an individual. Spasticity management strategies often differ by center. On one end of this strategy spectrum is a highly-interventional approach, characterized by treatments such as a selective dorsal rhizotomy (SDR), intrathecal baclofen pump (ITB), and antispasticity injections and medications. On the other end of the spectrum is a less interventional approach, involving minimal use of these treatments, and no SDR.

Methods and analysis A retrospectively-matched, multicenter study protocol is described that comprehensively compares the long-term outcomes of a highlyinterventional versus a minimally-interventional spasticity management strategy. We will analyze two groups of adults with spastic bilateral $\mathrm{CP}$ ( $\geq 21$ years). In one group are individuals who underwent an SDR between the ages of 4 and 10 years, along with ongoing spasticity management during childhood and adolescence. In the other group are individuals who received minimal spasticity management and did not undergo an SDR. Individuals with prolonged use of an intrathecal baclofen (ITB) pump will be excluded. The two groups will be matched for spasticity and other important clinical characteristics at baseline. This study design improves on many of the limitations found in the existing outcome literature.

Ethics and dissemination This study received necessary approval from the University of Minnesota and Western Institutional Review Boards. Results will be disseminated via peer-reviewed publications and conference presentations.

Trial registration number NCT03789786.

\section{INTRODUCTION}

While spasticity is the most common form of hypertonia in cerebral palsy (CP) and affects up to $80 \%$ of individuals with $\mathrm{CP},{ }^{12}$ strategies regarding spasticity management differ widely. Options range from focal injections

\section{Strengths and limitations of this study}

- Designed to assess outcomes of differing spasticity management strategies rather than an isolated surgical procedure.

- Cohort study using a retrospectively matched cerebral palsy control group.

- Multicentre recruitment strategy, allowing for more generalisable results

- Long-term follow-up and comprehensive outcome measures motivated by the International Classification of Functioning, Disability and Health framework.

- Due to the nature of long-term follow-up, a significant number of eligible participants will be lost to follow-up, which may result in sampling bias.

with small temporary effects, such as botulinum toxin or phenol injections, to global reversible approaches with varying effects, such as oral and intrathecal baclofen (ITB), to irreversible methods with large effects, such as selective dorsal rhizotomy (SDR). An SDR is a surgical procedure that largely normalises muscle spasticity ${ }^{3}$ and is the core of the highly interventional management approach described here. When performed during early childhood, spasticity reduction by means of an SDR aims to prevent or minimise future joint contracture, excess bony torsion, high energy cost and gait impairment, thereby improving function, comfort, pain and quality of life. While SDR has been repeatedly shown to largely eliminate spasticity immediately and sustainably, questions remain regarding the other hypothesised benefits SDR aims to achieve. ${ }^{4}$ Additionally, despite undergoing an SDR, many children and adolescents continue to receive ongoing antispasticity management. As a result of the 
uncertainty surrounding spasticity management, some clinicians take an aggressive approach to reducing spasticity, while others opt for minimal spasticity reduction management. No definitive evidence exists to support either of these strategies in ambulatory patients with bilateral spastic CP.

Both the unknown natural history of $\mathrm{CP}$ and the heterogeneity of symptoms make it difficult to measure and interpret the outcomes of spasticity management strategies. The quality of evidence from past outcomes research related to SDR, arguably the most studied treatment in this patient population, is poor. The literature is characterised by studies with small numbers of participants and poor study designs. Examples include studies lacking a proper control group or relying entirely on surveys, which are susceptible to bias and subjective impressions. ${ }^{5}{ }^{6}$ Short-term outcome studies show favourable results for SDR, including improvements from baseline in spasticity, range of motion across the lower extremities, gait impairment and function. However, long-term longitudinal studies suggest that many outcomes peak at 1-3 years post-SDR. ${ }^{47}$ More concerning is that most studies, both short and long term, fail to use a proper control group. This makes it difficult to differentiate improvements attributable to spasticity reduction from those due to growth and development, or other treatments, such as orthopaedic surgery. While an ITB pump is another global tool to manage spasticity, it is more often used in patients classified as Gross Motor Function Classification System levels III-V, ${ }^{8}$ and is outside the scope of work presented here.

Gillette Children's Specialty Healthcare recently performed a long-term pilot study on SDR that used a broad range of outcomes and included a control group (ie, No-SDR), identified using propensity score matching. ${ }^{9}$ The control group was composed of individuals with CP who met the clinical criteria for receiving an SDR at our centre. The results of this study showed that the No-SDR group had larger improvements in gait compared with the SDR group, but underwent significantly more treatments between baseline and follow-up. Low levels of pain and high quality of life were reported equally between the groups with no statistical difference. However, as a pilot study, participant numbers were small $(\mathrm{n}=35)$, and the SDR group was slightly younger $(\sim 1.5$ years $)$ and had more spasticity in two of five lower extremity muscles (hip adductors and rectus femoris) at baseline. Additionally, the 11 No-SDR participants went on to have significant spasticity management as part of standard of care, including 3 ITB pump placements, and an average of 22 anti-spastic injections. Because of these limitations, it was not possible to conclusively differentiate between outcomes that arose from SDR, other forms of spasticity reduction, orthopaedic surgery or growth and development. This ambiguity motivated the study design proposed here, whereby we aim to better isolate the effects of a highly interventional spasticity management strategy that includes, but is not limited to, SDR.
Given the current state of the literature, the potential benefits of spasticity management remain poorly understood. A randomised controlled trial followed by a longitudinal observation period would be an ideal study design, but the feasibility of and ethical issues associated with such a design make it impractical. Even if it were practical to randomise individuals to a sole procedure, such as an SDR, the results of such a study would not be generalisable to the broader CP population, who often undergo many treatments for spasticity and orthopaedic deformity. The present study has been designed to compare two ends of the spasticity management spectrum. On one end are individuals cared for under a highly interventional management philosophy for spasticity. These individuals had spasticity reduced at an early age via SDR, and maintained with ongoing management throughout their childhood and adolescent years. On the other end are individuals cared for under a minimally interventional strategy. Using a matched control group is an essential step towards assessing the risks and benefits of any management approach. Given the often-uniform management strategy within any given centre, finding an internal control group is challenging. A multicentre approach, composed of institutions with clinicians using varying strategies regarding spasticity management, offers a solution to this problem.

The focus of the present study is to compare a highly interventional spasticity management strategy, including an SDR at an early age, to a minimally interventional strategy. We do this because (1) SDR has the largest impact on spasticity levels, (2) SDR is a permanent procedure and (3) SDR candidacy is how eligible patients were selected and matched between centres. We will refer to the groups in our report as 'Yes-SDR' and ' No-SDR' . To be clear, as part of their standard of care, participants in the Yes-SDR group will most often have had additional spasticity management through focal injections or oral medications, and we will control for these treatments in the final analysis. Participants in the No-SDR group will have no history of an SDR, and will have had only minimal spasticity management, as will be defined in the protocol details described below. Use of an ITB pump was cause for exclusion from both groups, unless it only used temporarily (duration $<1$ year).

Our primary hypothesis is that as adults (age $\geq 21$ ), individuals in the Yes-SDR group, who underwent early, aggressive and ongoing spasticity management, will have significantly larger improvements in gait quality compared with a control group who underwent minimal spasticity management. Secondary hypotheses are that, at follow-up, the Yes-SDR group will have had less orthopaedic surgery and better spasticity, function, energy, pain and quality of life.

\section{METHODS AND ANALYSIS}

\section{Overall study design}

This is a multicentre cohort study including three medical centres: Gillette Children's Specialty Healthcare - St. Paul, MN (GIL), Shriners Hospitals for Children Spokane, WA (SPK) and Shriners Hospitals for Children - Salt Lake City, UT (SLC). Each centre will identify 
potential participants as outlined below. Participants are identified based on data collected retrospectively during a baseline routine clinical evaluation, including gait analysis and physical examination. Each centre will then apply additional inclusion criteria related to current age and treatment history. Eligible participants will be recruited to complete a variety of outcome measures at a single, prospective, long-term follow-up visit. Based on design, the final pool of participants should be matched at baseline for age and overall lower limb spasticity.

\section{Participants}

Two groups of participants will be recruited into this study.

- Yes-SDR group: individuals treated with an SDR at GIL.

- No-SDR group: individuals with history of minimal spasticity management and no history of SDR, recruited from SPK and SLC.

Because SDR is the centrepiece of the highly interventional approach practised at GIL, participant selection was designed so that participants at SPK and SLC met the GIL criteria for an SDR at their baseline visit. This was achieved through three steps detailed below. A fourth step outlines eligibility parameters applied at the time of long-term follow-up.

\section{Step 1: identify patients to be included in a lower limb spasticity} score distribution

The goal of step 1 was to find the distribution of untreated spasticity among patients at each centre. Each of the three participating centres then identified individuals meeting criteria for an SDR at GIL. These data came from a baseline clinical gait analysis; therefore, all individuals were ambulatory. Additional inclusion criteria and their rational were as follows:

- Bilateral CP: Patients with a diagnosis of diplegia, triplegia or quadriplegia were included. Children diagnosed with hemiplegia are not considered candidates for SDR at GIL and were excluded.

- Untreated spasticity: Individuals who had spasticity measured during a baseline examination and had no history of an SDR were included. Individuals with an indwelling ITB pump or recent focal antispastic injections were excluded to avoid skewing the spasticity distribution.

- Age between 3.5 and 10.5 years: This is the $5-95$ th percentile for age at baseline gait analysis prior to SDR at GIL.

Applying these criteria resulted in three baseline populations (one for each centre) of young patients with bilateral CP and currently untreated spasticity. The next step was generating a lower limb spasticity score.

Step 2: generate a baseline lower limb spasticity score distribution The goal of step 2 was to allow us to match patients for overall spasticity between centres. Matching historical lower limb spasticity across centres presents a challenge.
The three centres participating in this study have not always collected the same measures of spasticity. Furthermore, it is possible that there could be between-centre differences in how the same spasticity scales are scored. For example, a centre that has highly interventional spasticity management strategy may have a measurement bias skewed towards higher spasticity. To overcome this challenge, each centre developed an overall lower limb spasticity score. We then categorised potential participants as exhibiting mild, moderate or severe spasticity based on this spasticity score, and sampled individuals using these categories in a manner that reflected the distribution of spasticity among SDR-eligible patients at GIL. This approach assumed that patients seen at the three centres are random samples from the same underlying population of children with CP, and therefore, the actual spasticity distributions are equal between the centres. It is not possible to directly confirm this a priori; however, distributions of demographic, clinical and functional parameters for each centre's patient base will be evaluated and reported as part of the final results of the study to test this assumption.

At GIL and SPK, the spasticity score was defined as the mean of bilateral Ashworth scores for four muscles: hip adductors, hamstrings, vasti/rectus femoris and ankle plantar flexors. Individuals needed to have at least three of four spasticity measures on each side to have their data included. We made the assumption that missing one spasticity measure per limb still allowed for an unbiased summary of overall spasticity, while maintaining an adequate sample size.

At SLC, Ashworth scores were not always used to document spasticity. Therefore, the lower limb spasticity score at SLC was defined as a weighted average of up to five different bilateral spasticity measures: (1) mean modified Ashworth scores for at least three bilateral muscles: hamstrings, vasti/rectus femoris, ankle plantar flexors and ankle invertors, (2) Duncan Ely for rectus femoris, (3) beats of clonus, (4) difference between ankle initial stretch and final range of motion as a surrogate for ankle plantar flexor spasticity and (5) deep tendon reflex. All scores were scaled to be on a standardised $0-5$ range, where 5 was the higher severity for the measure. Modified Ashworth scores were weighted by a factor of two due to their relative importance compared with other measures
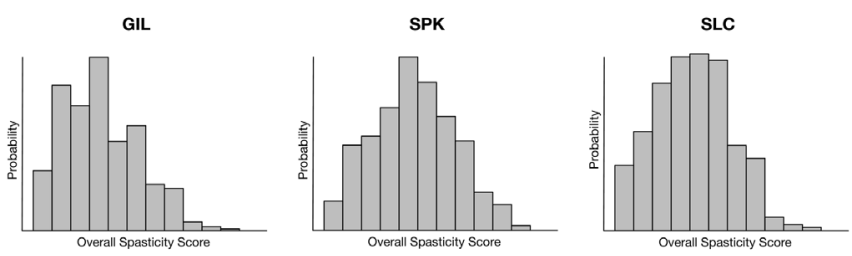

Figure 1 Distribution for overall spasticity of patients with cerebral palsy who had gait analysis in each centre. GIL, Gillette Children's Specialty Healthcare; SLC, Shriners Hospitals for Children in Salt Lake City; SPK, Shriners Hospitals for Children in Spokane. 
Yes-SDR

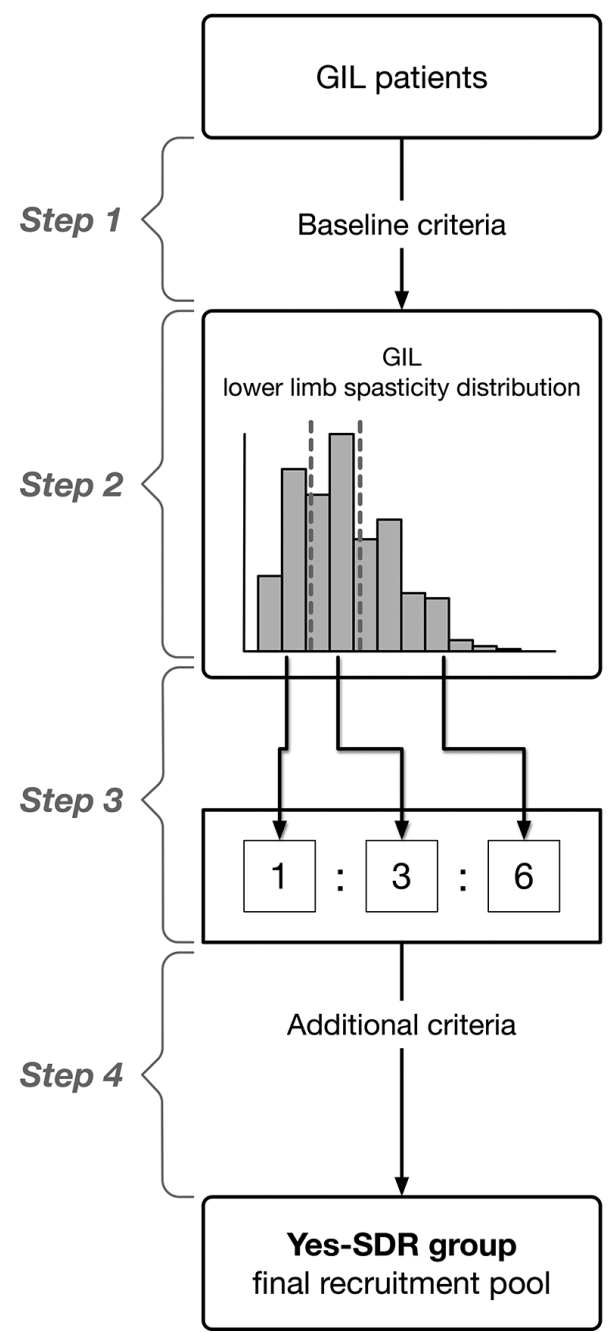

No-SDR

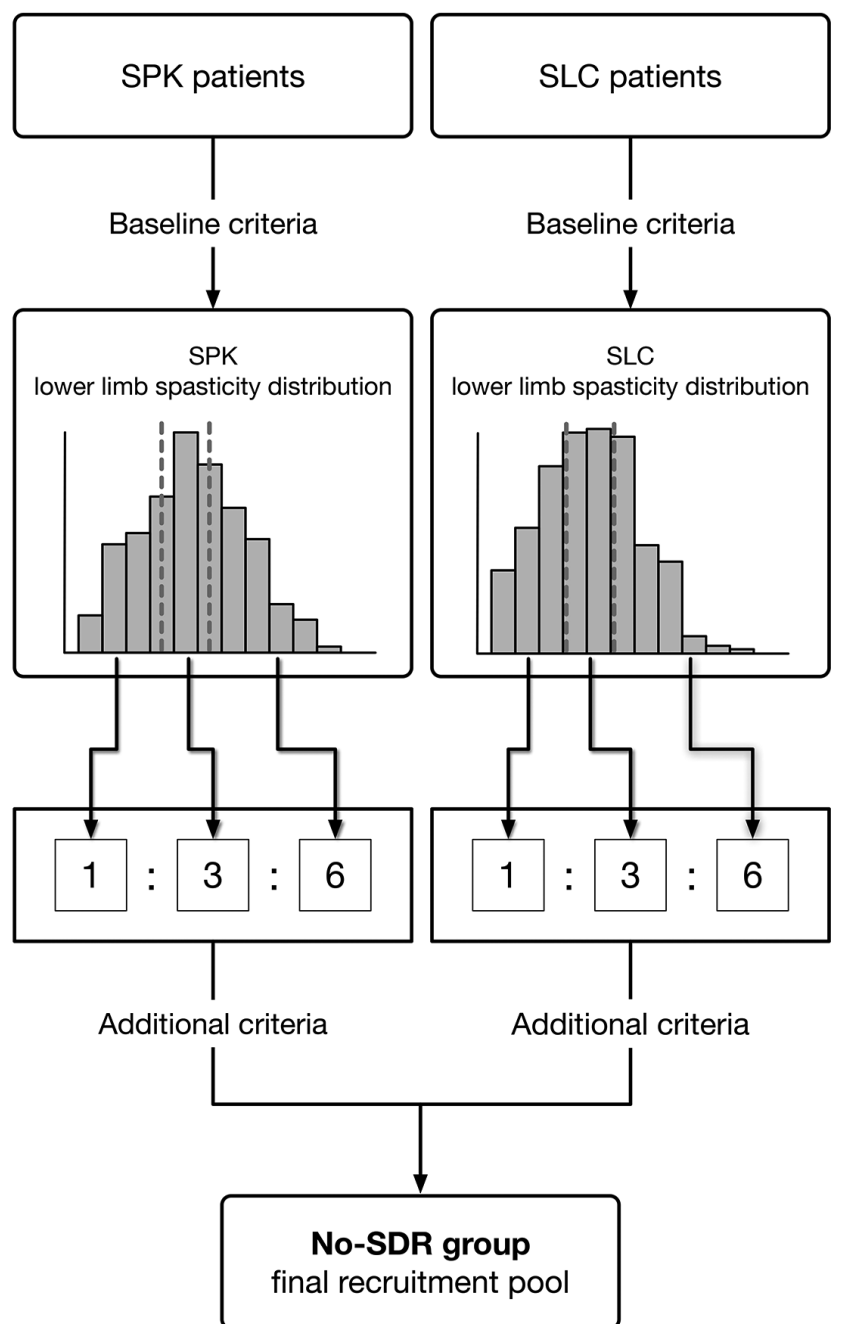

Figure 2 The selection process sites will follow in order to identify potential participants in the Yes-SDR and No-SDR groups. This sampling procedure should lead to matched groups at baseline. GIL, Gillette Children's Specialty Healthcare; SDR, selective dorsal rhizotomy; SLC, Shriners Hospitals for Children in Salt Lake City; SPK, Shriners Hospitals for Children in Spokane.

(ie, more direct measure of spasticity and utilisation by GIL and SPK). See online supplementary appendix 1 for additional detail. After accounting for differences in spasticity measures, a distribution for overall spasticity was generated for each centre (figure 1).

\section{Step 3: sampling from centre's overall lower limb spasticity score} distribution

The goal of step 3 was to design a sampling strategy for SPK and SLC so that the final pool of study participants will have a baseline spasticity distribution matching that of individuals who received an SDR at GIL. An evaluation of historical spasticity scores for patients at GIL showed that children receiving an SDR were drawn from the overall distribution in an approximate ratio of $10 \%$ from the lowest tertile (mild), $30 \%$ from the middle tertile (moderate) and $60 \%$ from the highest tertile (severe) of lower limb spasticity. As a result, during recruitment, both SPK and SLC will sample from their populations in a 1:3:6 ratio (ie, $10 \%$ mild spasticity, $30 \%$ with moderate spasticity and $60 \%$ with severe spasticity). This should result in SDR-eligible patients at SPK and SLC matching SDR patients from GIL for age and spasticity at baseline (figure 2). Note that age limits were enforced in Step 1.

Step 4: criteria for final recruitment pool at long-term follow-up Following targeted sampling of individuals with mild, moderate and severe spasticity profiles, additional criteria will be applied to eligible participants to form the final recruitment pool of adults for long-term follow-up.

\section{For participants in the Yes-SDR group}

- At least 21 years old at the time of long-term follow-up.

- At least 10 years post-SDR. 
- No history of a health event likely to impact outcome measures and unlikely to be related to spasticity or hypotonia (eg, pregnancy at the time of follow-up).

For participants in the No-SDR group

- Meet same criteria as SDR group, except for history of SDR.

\section{Additional criteria to ensure minimal spasticity management}

- No history of ITB pump implantation for more than 1 year.

- If there is a history of ITB pump for less than 1 year, then ITB pump must have been explanted at least 6 months prior to long-term follow-up.

- No history of oral baclofen use for more than 1 year.

- If there is a history of oral baclofen use for less than 1 year, then oral baclofen use must have been discontinued at least 6 months prior to long-term follow-up.

- No more than three instances of spasticity management by means of focal injection.

- If there is a history of three or fewer episodes of spasticity management by focal injection, then the most recent injection must have occurred at least 8 weeks prior to long-term evaluation.

\section{Data collection}

At the single long-term follow-up visit, a variety of comprehensive outcomes measures will be collected. Outcomes will span International Classification of Functioning, Disability and Health (ICF) domains. ${ }^{10}$

1. Gait Quality (ICF domain: body function and structure):

a. Gait Deviation Index (GDI). ${ }^{11}$

b. Dynamic knee and ankle range of motion from gait data (sagittal kinematics).

c. Walking speed.

d. Energy expenditure assessment. ${ }^{12}$

e. Dynamic motor control during gait. ${ }^{13} 14$

2. Lower Extremity Clinical Profile (ICF domain: body function and structure):

a. Spasticity measured by physical examination. ${ }^{15} 16$

b. Static hip, knee and ankle range of motion from physical examination.

c. Muscle strength. ${ }^{17}$

3. Gross Motor Function (ICF domain: body function and structure):

a. Gross Motor Function Measure, dimension D: standing and Dimension E: walking, running and jumping. ${ }^{18}$

b. Gillette Functional Assessment Questionnaire. ${ }^{19}$

c. Functional Mobility Scale. ${ }^{20}$

d. Gross Motor Function Classification System. ${ }^{21}$

4. Activity and participation, quality of life, pain and comfort: (ICF domains: activity and participation).

a. Participation enfranchisement. ${ }^{22}$

b. Satisfaction with Life Scale. ${ }^{23}$

c. Abbreviated WHO Quality of Life Scale. ${ }^{24}$ d. Multiple Sclerosis Spasticity Scale. ${ }^{25}$

5. Treatment history

a. Incidence of surgery and focal antispastic injections.

\section{Data analysis}

We intend to recruit 40 individuals into the Yes-SDR group from GIL and 40 individuals into the No-SDR group (20-SPK, 20-SLC). This number is estimated from a power analysis, based on mean change in GDI as our primary outcome measure, using an effect size of 0.75 , a 0.05 significance level and a power of 0.9. Mean and SD of change scores were estimated from the results of the Gillette pilot study. ${ }^{9}$ We chose a medium to large effect size due to the aggressive and irreversible nature of SDR, and the expectation that a significant benefit should be gained. Our study is designed to measure two types of differences: (1) changes from baseline to follow-up within groups and (2) differences between Yes-SDR and No-SDR groups at follow-up, across a variety of outcomes measures. The proposed study design should result in Yes-SDR and No-SDR groups matched at baseline. Baseline variables related to age, spasticity, gait impairment and overall function will be tested for equivalence, and, if baseline variables are not matched, these differences will be accounted for in the statistical analysis.

\section{DISCUSSION}

Large gaps in knowledge remain regarding the value of a highly interventional or a minimally interventional spasticity management strategy. We expect that the results of our study will clarify the long-term impact that varying levels of spasticity management have on ambulatory children with bilateral spastic CP.

Our new multicentre study design offers several improvements over previously published studies of SDR outcomes. We expect the results to be more useful to clinicians and patients, since they assess outcomes of differing spasticity management strategies rather than isolated surgical procedures. Collaboration between centres who use different spasticity management strategies will allow us to compare the outcomes of highly interventional spasticity management with those of a control group who underwent minimal spasticity management. By including multiple outcomes domains from the ICF model, we will acquire a comprehensive understanding of the long-term benefits of different spasticity management strategies. For example, do outcome advantages in one domain, such as body structure and function, translate into outcome advantages in another, such as activity and participation? Lastly, by tracking frequency of treatments that occur between baseline and follow-up, we can measure any cost-effectiveness differences that exist between the two management strategies.

Although the methods of this study have been carefully designed, there are still known limitations. Potential participants will be identified based on data in the medical record that are many years old. Therefore, 
participants may be lost to follow-up and difficult to enrol. Our eligible participant numbers and enrolment numbers will be reported for transparency. Because this is not a randomised controlled trial, confounding variables may exist. While observed confounders can be adjusted for statistically, unobserved confounders cannot. Finally, there may be differences in orthopaedic treatment strategies between the centres. These will be considered when evaluating frequency and cost of treatments occurring between baseline and follow-up.

\section{Dissemination}

This study will be disseminated via peer-reviewed publications and conference presentations.

\section{Patient and public involvement}

Patients were not explicitly involved in the design of this research work; however, outcomes thought to be priorities in patients' lives, as based on the ICF guidelines, were picked in order to critically and comprehensively examine outcomes of spasticity management. A newsletter summarising study findings will be sent to all participants after the completion of the study.

\section{Author affiliations}

${ }^{1}$ Gillette Children's Specialty Healthcare, Center for Gait and Motion Analysis, St. Paul, Minnesota, USA

${ }^{2}$ University of Minnesota Twin Cities, Orthopaedic Surgery, Minneapolis, Minnesota, USA

${ }^{3}$ Shriners Hospitals for Children Salt Lake City, Motion Analysis Center, Salt Lake City, Utah, USA

${ }^{4}$ University of Utah, Department of Orthopaedics, Salt Lake City, Utah, USA

${ }^{5}$ Walter E. Griffin and Agnes M Griffin Motion Analysis Center, Shriners Hospital for Children Spokane, Spokane, Washington, USA

Acknowledgements We would like to acknowledge Elizabeth A Duffy for her contribution to this study.

Contributors MHS, BM, MLM and MEM designed the study. MHS was in charge of the statistical analysis. MEM and BP-JC wrote the draft of the manuscript. All authors revised the manuscript for important intellectual content and provided approval of the final version to be published.

Funding This work was supported by the Gait and Motion Outcomes Fund of the Gillette Children's Foundation.

Competing interests None declared.

Patient consent for publication Not required.

Ethics approval This study received necessary approval from University of Minnesota and Western Institutional Review Boards.

Provenance and peer review Not commissioned; externally peer reviewed.

Open access This is an open access article distributed in accordance with the Creative Commons Attribution Non Commercial (CC BY-NC 4.0) license, which permits others to distribute, remix, adapt, build upon this work non-commercially, and license their derivative works on different terms, provided the original work is properly cited, appropriate credit is given, any changes made indicated, and the use is non-commercial. See: http://creativecommons.org/licenses/by-nc/4.0/.

\section{REFERENCES}

1. Howard J, Soo B, Graham HK, et al. Cerebral palsy in Victoria: motor types, topography and gross motor function. J Paediatr Child Health 2005;41:479-83.

2. Christensen D, Van Naarden Braun K, Doernberg NS, et al. Prevalence of cerebral palsy, co-occurring autism spectrum disorders, and motor functioning - Autism and Developmental Disabilities Monitoring Network, USA, 2008. Dev Med Child Neurol 2014;56:59-65.

3. Trost JP, Schwartz MH, Krach LE, et al. Comprehensive short-term outcome assessment of selective dorsal rhizotomy. Dev Med Child Neurol 2008;50:765-71.

4. Tedroff K, Löwing K, Åström E. A prospective cohort study investigating gross motor function, pain, and health-related quality of life 17 years after selective dorsal rhizotomy in cerebral palsy. Dev Med Child Neurol 2015;57:484-90.

5. Park TS, Edwards C, Liu JL, et al. Beneficial effects of childhood selective dorsal rhizotomy in adulthood. Cureus 2017;9:e1077.

6. Park TS, Liu JL, Edwards C, et al. Functional outcomes of childhood selective dorsal rhizotomy 20 to 28 Years Later. Cureus 2017;9:e1256.

7. Langerak NG, Lamberts RP, Fieggen AG, et al. A prospective gait analysis study in patients with diplegic cerebral palsy 20 years after selective dorsal rhizotomy. J Neurosurg Pediatr 2008;1:180-6.

8. Dan B, Motta F, Vles JS, et al. Consensus on the appropriate use of intrathecal baclofen (ITB) therapy in paediatric spasticity. Eur $J$ Paediatr Neurol 2010;14:19-28.

9. Munger ME, Aldahondo N, Krach LE, et al. Long-term outcomes after selective dorsal rhizotomy: a retrospective matched cohort study. Dev Med Child Neurol 2017;59:1196-203.

10. Organization WH. International classification of functioning, disability and health: ICF. Geneva: World Health Organization, 2001.

11. Schwartz MH, Rozumalski $A$. The gait deviation index: a new comprehensive index of gait pathology. Gait Posture 2008;28:351-7.

12. Schwartz MH, Koop SE, Bourke JL, et al. A nondimensional normalization scheme for oxygen utilization data. Gait Posture 2006;24:14-22

13. Steele KM, Rozumalski A, Schwartz MH. Muscle synergies and complexity of neuromuscular control during gait in cerebral palsy. Dev Med Child Neurol 2015;57:1176-82.

14. Schwartz MH, Rozumalski A, Steele KM. Dynamic motor control is associated with treatment outcomes for children with cerebral palsy. Dev Med Child Neurol 2016;58:1139-45.

15. Bohannon RW, Smith MB. Interrater reliability of a modified Ashworth scale of muscle spasticity. Phys Ther 1987;67:206-7.

16. Ashworth B. Preliminary trial of carisoprodol in multiple sclerosis. Practitioner 1964;192:540-2.

17. Lovett RW, Martin EG. Certain aspects of infantile paralysis: with a description of a method of muscle testinG. JAMA 1916;LXVI:729-33.

18. Russell DJ, Avery LM, Rosenbaum PL, et al. Improved scaling of the gross motor function measure for children with cerebral palsy: evidence of reliability and validity. Phys Ther 2000;80:873-85.

19. Novacheck TF, Stout JL, Tervo R. Reliability and validity of the Gillette Functional Assessment Questionnaire as an outcome measure in children with walking disabilities. J Pediatr Orthop 2000;20:75-81.

20. Graham HK, Harvey A, Rodda J, et al. The Functional Mobility Scale (FMS). J Pediatr Orthop 2004;24:514-20.

21. Palisano $R$, Rosenbaum $P$, Walter $S$, et al. Development and reliability of a system to classify gross motor function in children with cerebral palsy. Dev Med Child Neurol 1997;39:214-23.

22. Heinemann AW, Lai JS, Magasi S, et al. Measuring participation enfranchisement. Arch Phys Med Rehabil 2011;92:564-71.

23. Diener E, Emmons RA, Larsen RJ, et al. The satisfaction with life scale. J Pers Assess 1985;49:71-5.

24. World Health Organization. WHOQOL-BREF: introduction, administration, scoring and generic version of the assessment: field trial version. Geneva: World Health Organization, 1996.

25. Hobart JC, Riazi A, Thompson AJ, et al. Getting the measure of spasticity in multiple sclerosis: the Multiple Sclerosis Spasticity Scale (MSSS-88). Brain 2006;129:224-34. 